\title{
Risk of Recurrence in Surgically Resected Stage I Adenocarcinoma of the Lung: Histopathologic and Immunohistochemical Analysis
}

\author{
Sukki Cho $\cdot$ Sook-Whan Sung $\cdot$ Sanghoon Jheon $\cdot$ \\ Jin-Haeng Chung
}

Received: 30 May 2008/Accepted: 1 September 2008/Published online: 4 October 2008

(C) Springer Science+Business Media, LLC 2008

\begin{abstract}
Study Objectives Stage I adenocarcinoma of the lung is the most common type of lung cancer. A better understanding of the histopathology and molecular biology of lung cancer might improve the capability to predict the outcome for any individual patient. The purpose of this study was to evaluate several histopathologic and molecular markers in order to assess their prognostic value in stage I adenocarcinoma. Methods Fifty-five patients at the Seoul National University Bundang Hospital were enrolled in the study. Histopathologic factors and molecular markers were selected. Results Multivariate analysis demonstrated that necrosis, lymphatic vessel invasion, E-cadherin, p53, and $\mathrm{Ki}-67$ emerged as independent prognostic factors of recurrence. When patients were grouped according to low or high risk according to the number of factors involved, the difference in disease-free survival between these groups was statistically significant. Conclusions In resected stage I adenocarcinoma, necrosis, lymphatic vessel invasion,
\end{abstract}

S. Cho $\cdot$ S.-W. Sung $(\bowtie) \cdot$ S. Jheon

Department of Thoracic and Cardiovascular Surgery,

Seoul National University Bundang Hospital, 300 Gumi-dong,

Bundang-gu, Seongnam-si, Gyeonggi-do 463-707,

South Korea

e-mail: swsung@ snubh.org

S.-W. Sung · S. Jheon

Department of Thoracic and Cardiovascular Surgery,

Seoul National University College of Medicine,

Seoul, South Korea

J.-H. Chung

Department of Pathology, Seoul National University Bundang Hospital, Gyeonggi-do, South Korea

J.-H. Chung

Department of Pathology, Seoul National University College

of Medicine, Seoul, Korea
E-cadherin, and p53 have been identified as independent predictors of disease-free survival.

Keywords Lung cancer $\cdot$ Stage I - Adenocarcinoma · Prognostic factors $\cdot$ Recurrence

\section{Introduction}

Lung cancer is the most common cause of mortality worldwide. Non-small-cell lung cancer (NSCLC) accounts for approximately $80 \%$ of lung cancer cases [1], and pathologic stage $\mathrm{I}$ is the fastest growing segment as found by the use of low-dose computed tomography (LDCT) for screening. Despite the potential benefits of surgical resection, patients with pathologic stage I lung cancer display marked variability in recurrence and survival. Even after complete resection, the 5-year survival rate of stage I patients is only $55-72 \%$, predominantly as a result of the development of distant metastases [2,3]. Variation in survival largely reflects heterogeneity in tumor biology, with some tumors having more aggressive growth and greater metastatic potential than others; therefore, tumor stage alone is no longer the primary determinant of prognosis for these patients. New prognostic factors need to be identified to help clinicians better assess the probability of survival and optimize therapeutic strategies for each individual patient with pathologic stage I lung cancer. A better understanding of the molecular biology of NSCLC may help predict the outcome for individual patients, which in turn would facilitate the definition of subgroups of patients according to prognosis [4]. Several studies have already demonstrated possible prognostic roles for several biological factors in NSCLC and have found helpful tools for identifying patients with poor prognosis [5-8]. As a result, 
the stratification of patients, without lymph node involvement, according to prognostic risk might aid in selecting a group of high-risk patients who would benefit from adjuvant therapy.

The purpose of this study was to evaluate several histopathologic variables and a panel of molecular markers in order to assess their prognostic value and their combined effects on recurrence in patients with resected stage I NSCLC.

\section{Materials and Methods}

\section{Patient Characteristics}

Between June 2003 and August 2005, 55 patients (31 male, 24 female) at the Seoul National University Bundang Hospital (SNUBH) with resected stage I adenocarcinoma were enrolled in the study. All patients underwent potentially curative surgery consisting of lobectomy $(n=52)$ or segmentectomy $(n=3)$ and complete mediastinal lymph node dissection. None of the patients had neoadjuvant therapy. Patients who died within 1 month after surgery were excluded from the study to avoid the bias of perioperative mortality. This study was approved by the Institutional Review Board of SNUBH.

The age of the patients ranged from 40 to 81 years (mean $=63.4$ years). Postsurgical pathologic TNM staging was determined according to the guidelines of the American Joint Cancer Committee (AJCC). There were 28 cases of stage IA (T1NOM0) and 27 cases of stage IB (T2NOM0). Follow-up data on the study population were obtained by direct contact. Follow-up occurred at 3-month intervals for the initial 3 years and at 4-month intervals thereafter. Recurrences were detected by computed tomography scans or by positron emission tomography and, if necessary, confirmed by pathologic examination of biopsy specimens. Patients were categorized as alive with evidence of disease or alive without disease. No patient in this series died of cancer-unrelated causes. Time from the date of the operation to the date of follow-up or death was recorded. Local recurrence is defined as tumor recurrence at the ipsilateral lung or lymph node, and distant recurrence is defined as tumor recurrence at the contralateral lung or lymph node and a distant organ such as the liver, brain, or bone.

\section{Pathologic Criteria}

Tumor samples were fixed in $10 \%$ buffered formalin, dehydrated, and embedded in paraffin. Then, 4- $\mu$ l-thick sections were cut and stained with hematoxylin and eosin. Pathologic features were classified according to the histologic criteria of the World Health Organization. The degree of differentiation was divided into three groups: well, moderate, and poor. Tumor vascular invasion (absent vs. present) and lymphatic vessel invasion (absent vs. present) were defined as the identification of emboli composed of neoplastic cell aggregates within the tumor vessel and lymphatic vessel, respectively. Tumor necrosis (negative, $<10 \%$; positive, $\geq 10 \%$ ) and visceral pleural invasion (absent vs. present) were noted. Tumor size $(<3 \mathrm{~cm}$ vs. $\geq 3 \mathrm{~cm}$ ) was also recorded.

Immunohistochemical Methods

Briefly, tissues were deparaffinized in xylene and rehydrated in graded alcohols and water. Endogenous peroxidase was blocked by soaking in $3 \% \mathrm{H}_{2} \mathrm{O}_{2}$ at $45^{\circ} \mathrm{C}$ for $4 \mathrm{~min}$. The slides were microwaved in citrate buffer $(2.1 \mathrm{~g} /$ $\mathrm{L}, \mathrm{pH}$ 6.0) at $120^{\circ} \mathrm{C}$ for 15 min to unmask the antigen and were then treated with a protein-blocking reagent before incubation at $4{ }^{\circ} \mathrm{C}$ overnight with primary antibodies at a 1:50 dilution, as recommended by the manufacturer. After extensive washing, the sections were incubated with biotinylated anti-mouse immunoglobulin antibodies (Zymed Essence Co. San Francisco, CA) at a 1:20 dilution at room temperature for $10 \mathrm{~min}$ and subsequently with streptavidinbiotin peroxidase complexes at a 1:25 dilution. Reaction products were visualized by immersing the slides in 3,3'diaminobenzidine tetrahydrochloride. Counterstaining was performed with hematoxylin. All series included positive and negative controls. Negative controls were prepared by omitting the primary antibodies, and known positive controls were included in each run.

\section{Evaluation of Immunohistochemical Data}

Glut-1 expression was assessed as the percentage of the area positive for Glut-1 staining and was graded as 1 (positive area $<25 \%), 2(\geq 25 \%,<50 \%), 3(\geq 50 \%,<75 \%$ ), and $4(\geq 75 \%)$. Expression of p53 was determined in tumor cells according to the degree of brown nuclear staining; specimens were considered negative when nuclear staining was less than $10 \%$ and positive when greater than $10 \%$. The Ki-67 labeling index (LI) was scored by counting at least 1000 neoplastic cells in 10-20 fields representing different tumor areas. All stained nuclei were considered positive regardless of the staining intensity, and specimens with up to $20 \%$ positively stained tumor cells were considered to have a low proliferative index, and cases with more than $20 \%$ positively stained cells were considered to have a high proliferative index. Regarding p16 expression, only nuclear staining was considered to be positive and any cytoplasmic staining present was considered to be nonspecific. Loss of p16 was defined as less than $10 \%$ of 
nuclear staining. Cyclin D1 was considered overexpressed when $10 \%$ or more of cell nuclei were stained. In Rb expression, only nuclear staining was considered to be positive and any cytoplasmic staining present was considered to be nonspecific. Rb loss was defined as less than $10 \%$ of nuclear staining.

E-cadherin was regarded as lost $(<25 \%$ of cytoplasmic staining) or preserved $(\geq 25 \%)$. Epithelial growth factor receptor (EGFR) expression was assessed as intensity of staining from 0 to 3 and graded as normal (0 and 1) or overexpressed (2 and 3 ).

\section{Statistical Analysis}

The $\chi^{2}$ test and Fisher's exact test were used to analyze the association between histopathologic variables, molecular variables, and recurrence. The time to relapse was defined as the period from the date of surgery to the date when relapse was diagnosed. Specific time-to-recurrence curves were plotted using the Kaplan-Meier method, whereas the log-rank test was used to assess the statistical significance of differences between groups. Multivariate analyses were performed using the Cox proportional hazards model to identify independent prognostic factors. The criterion for significance was $p<0.05$.

\section{Results}

\section{Histopathology}

The average tumor size was $25.9 \pm 11.9 \mathrm{~mm}$ (range $=8-$ $75 \mathrm{~mm})$, and $15(27.3 \%)$ patients had a tumor size greater than $30 \mathrm{~mm}$. Twenty-five $(45.5 \%)$ tumors were well differentiated, $19(34.5 \%)$ were moderately differentiated, and $11(20.0 \%)$ were poorly differentiated. Ten $(18.5 \%)$ tumors showed necrosis, vascular invasion occurred in $3(5.6 \%)$ tumors, lymphatic vessel invasion occurred in 13 (24.1\%), and visceral pleural invasion occurred in 20 (36.4\%). Eight (14.5\%) patients had tumors of at least $3 \mathrm{~cm}$ as well as visceral pleural invasion tumors (Table 1).

\section{Immunohistochemical Staining}

Glut-1 expression was detected in 46 patients; $32(58.2 \%)$ patients were grade $1,3(5.5 \%)$ were grade $2,6(10.9 \%)$ were grade 3 , and $5(9.1 \%)$ were grade 4 . Positivity of p53 was found in $15(27.3 \%)$ patients. Expression of Ki-67 was observed in all patients, with $38(69.1 \%)$ having a low LI and $17(30.9 \%)$ having a high LI. There was a loss of p16 in $15(27.3 \%)$ cases and a loss of $\mathrm{Rb}$ in $18(32.7 \%)$ cases. Cyclin D1 was overexpressed in 43 (78.2\%) cases, Ecadherin was lost in $7(13.0 \%)$ cases, and EGFR was overexpressed in $24(43.6 \%)$ cases (Table 2).
Table 1 Histopathologic findings in 55 resected stage I adenocarcinoma cases

\begin{tabular}{ll}
\hline Characteristics & No. of patients $(\%)$ \\
\hline T stage & $28(50.9)$ \\
1 & $27(49.1)$ \\
2 & \\
Tumor size & $40(72.7)$ \\
$\leq 3$ & $15(27.3)$ \\
$>3$ & \\
Differentiation & $25(45.5)$ \\
Well & $19(34.5)$ \\
Moderate & $11(20.0)$ \\
Poor & \\
Necrosis & $45(81.5)$ \\
Absent & $10(18.5)$ \\
Present & \\
Visceral pleural invasion & $35(63.6)$ \\
Absent & $20(36.4)$ \\
Present & \\
Vascular invasion & $52(94.4)$ \\
Absent & $3(5.6)$ \\
Present & \\
Lymphatic vessel invasion & \\
Absent & $13(24.1)$ \\
Present &
\end{tabular}

Association Between Histopathologic and Molecular Factors

Glut-1 expression was significantly high in several factors such as positive p53 $(p=0.028)$, high LI Ki-67 ( $p=$ $0.001)$, and necrosis $(p=0.001)$. The positivity of $\mathrm{p} 53$ was correlated with high LI Ki-67 $(p=0.007)$ and vascular invasion $(p=0.044)$. A high LI of Ki-67 was correlated with necrosis $(p=0.001)$ and cyclin D1 $(p=0.030$, no expression). Loss of $\mathrm{Rb}$ was more frequent in patients with T2 stage tumors ( $p=0.015)$, with visceral pleural invasion $(p=0.001)$, and with cyclin D1 ( $p=0.001$, no expression).

\section{Recurrence}

Median follow-up was 28.9 months and the 3-year diseasefree survival rate was $77.7 \%$, with a mean disease-free time of 37.9 months. Twelve patients $(21.8 \%)$ had recurrent disease and six patients $(10.9 \%)$ died from disease-related causes. Three of the 12 patients had local recurrence and nine patients had distant recurrence. Of the 9 patients with distant metastasis, 3 had bone metastasis, 3 had recurrence at the contralateral lung, 1 at the liver, 1 at the brain, and 1 at multiple sites. Table 3 shows that histopathologic factors, including differentiation $(p=0.035)$, size $(p=0.046)$, 
Table 2 Immunohistochemical staining in 55 resected stage I adenocarcinoma cases

\begin{tabular}{|c|c|}
\hline Characteristics & No. of patients (\%) \\
\hline \multicolumn{2}{|l|}{ p16 } \\
\hline Loss & $15(27.3)$ \\
\hline Preserved & $40(72.7)$ \\
\hline \multicolumn{2}{|l|}{$\mathrm{Rb}$} \\
\hline Loss & $18(32.7)$ \\
\hline Preserved & $37(67.3)$ \\
\hline \multicolumn{2}{|l|}{ Cyclin D1 } \\
\hline Normal & $12(21.8)$ \\
\hline Overexpressed & $43(78.2)$ \\
\hline \multicolumn{2}{|l|}{ E-cadherin } \\
\hline Loss & $7(13.0)$ \\
\hline Preserved & $48(87.0)$ \\
\hline \multicolumn{2}{|l|}{ p53 } \\
\hline Negative & $40(72.7)$ \\
\hline Positive & $15(27.3)$ \\
\hline \multicolumn{2}{|l|}{ Glut- $1^{\mathrm{a}}$} \\
\hline 1 & $32(58.2)$ \\
\hline 2 & $3(5.5)$ \\
\hline 3 & $6(10.9)$ \\
\hline 4 & $5(9.1)$ \\
\hline \multicolumn{2}{|l|}{ Ki-67 } \\
\hline Low LI & $38(69.1)$ \\
\hline High LI & $17(30.9)$ \\
\hline \multicolumn{2}{|l|}{ EGFR } \\
\hline Normal & $31(56.4)$ \\
\hline Overexpressed & 24 (43.6) \\
\hline
\end{tabular}

$\mathrm{LI}=$ labeling index

a According to the positive area: $1=<25 \%, 2=\geq 25 \%$ and $<50 \%$, $3=\geq 50 \%$ and $<75 \%, 4=\geq 75 \%$

lymphatic vessel invasion $(p=0.017)$, necrosis $(p=$ $0.033)$, and molecular markers consisting of $\mathrm{p} 53$ positivity $(p=0.046)$ and E-cadherin loss $(p=0.036)$, were significantly associated with recurrence.

Table 4 lists the results of univariate analysis for the prognostic significance of each histopathologic factor and molecular marker in relation to the time to recurrence. The log-rank test showed that the $\mathrm{T}$ factor, necrosis, differentiation, lymphatic vessel invasion, E-cadherin loss, positive p53, and EFGR overexpression were prognostic factors (Fig. 1). Multivariate analysis using the Cox proportional hazards regression model, showed that necrosis, lymphatic vessel invasion, E-cadherin, p53, and Ki-67 were independent prognostic factors of recurrence (Table 4).

To further stratify patients, prognostic factors, including necrosis, lymphatic vessel invasion, E-cadherin, and p53, which were prognostic factors in both univariate and multivariate analyses, were chosen. Patients were then
Table 3 Recurrence according to histologic factors and molecular markers

\begin{tabular}{|c|c|c|c|}
\hline Characteristics & $\begin{array}{l}\text { No. of } \\
\text { patients }\end{array}$ & Recurrence & $p$ value \\
\hline Age (years) & & & 0.514 \\
\hline$<65$ & 25 & 4 & \\
\hline$\geq 65$ & 30 & 8 & \\
\hline Gender & & & 0.615 \\
\hline Male & 31 & 6 & \\
\hline Female & 24 & 6 & \\
\hline $\mathrm{T}$ stage & & & 0.055 \\
\hline 1 & 28 & 3 & \\
\hline 2 & 27 & 9 & \\
\hline Tumor size & & & 0.046 \\
\hline$\leq 3$ & 40 & 6 & \\
\hline$>3$ & 15 & 6 & \\
\hline Differentiation & & & 0.035 \\
\hline Well & 25 & 1 & \\
\hline Moderate & 19 & 5 & \\
\hline Poor & 11 & 6 & \\
\hline Necrosis & & & 0.033 \\
\hline Absent & 45 & 7 & \\
\hline Present & 10 & 5 & \\
\hline Visceral pleural invasion & & & 0.174 \\
\hline Absent & 33 & 5 & \\
\hline Present & 20 & 7 & \\
\hline Vascular invasion & & & 0.102 \\
\hline Absent & 51 & 9 & \\
\hline Present & 3 & 2 & \\
\hline $\begin{array}{l}\text { Lymphatic vessel } \\
\text { invasion }\end{array}$ & & & 0.017 \\
\hline Absent & 42 & 6 & \\
\hline Present & 13 & 6 & \\
\hline p16 & & & 0.274 \\
\hline Loss & 15 & 5 & \\
\hline Preserved & 40 & 7 & \\
\hline $\mathrm{Rb}$ & & & 0.960 \\
\hline Loss & 18 & 4 & \\
\hline Preserved & 37 & 8 & \\
\hline Cyclin D & & & 0.429 \\
\hline Normal & 12 & 4 & \\
\hline Overexpressed & 43 & 8 & \\
\hline E-cadherin & & & 0.036 \\
\hline Loss & 7 & 4 & \\
\hline Preserved & 47 & 8 & \\
\hline $\mathrm{p} 53$ & & & 0.046 \\
\hline Negative & 40 & 6 & \\
\hline Positive & 15 & 6 & \\
\hline Glut- $1^{\mathrm{a}}$ & & & 0.431 \\
\hline 1 & 32 & 6 & \\
\hline 2 & 3 & 1 & \\
\hline
\end{tabular}


Table 3 continued

\begin{tabular}{llll}
\hline Characteristics & $\begin{array}{l}\text { No. of } \\
\text { patients }\end{array}$ & Recurrence & $p$ value \\
\hline 3 & 6 & 3 & \\
4 & 4 & 1 & 1.000 \\
Ki-67 & & & \\
$\quad$ Low LI & 38 & 8 & 0.421 \\
$\quad$ High LI & 17 & 4 & \\
EGFR & & & \\
$\quad$ Normal & 31 & 5 & \\
$\quad$ Overexpressed & 24 & 7 & \\
\hline
\end{tabular}

$\mathrm{LI}=$ labeling index

a According to the positive area: $1=<25 \%, 2=\geq 25 \%$ and $<50 \%$, $3=\geq 50 \%$ and $<75 \%, 4=\geq 75 \%$

divided into two groups according to the number of risk factors present. The low-risk group $(n=44)$ had fewer than two risk factors and the high-risk group $(n=11)$ had two or more risk factors. Disease-free survival rates at 3 years were $88 \pm 5 \%$ for the low-risk group and $36 \pm 14 \%$ for the high-risk group ( $p<0.05$ by the logrank test) (Fig. 2).

\section{Discussion}

The prognosis for patients with cancer is divided into tumor-, patient-, and environment-related factors and a better understanding of the biology of tumors should provide useful information for predicting clinical outcomes and for individualizing treatment [9]. Because lung cancer is a heterogeneous disease resulting from the acquisition of multiple somatic mutations, the prognostic role of histopathologic and molecular markers is difficult to determine [9]. Both the differentiation of patients with a high risk of early recurrence and the identification of patients who may respond to adjuvant therapy are topics of great interest and have brought about intense study in this new era of pharmocogenomics. Although patients with stage I NSCLC fare significantly better than those with more advanced disease, a significant fraction of stage I patients experience disease recurrence and die after curative resection. Stratification of patient prognosis using only the TNM staging system does not allow one to differentiate patients with stage I lung cancer who are and are not at risk of tumor recurrence.

The role of chemotherapy after surgery for stage I disease remains a subject of debate. CALGB9633, a randomized trial that evaluated the effects of adjuvant chemotherapy, showed a statistically significant effect in favor of adjuvant chemotherapy for failure-free survival and overall survival for tumors larger than $4 \mathrm{~cm}$ [10]. In Japan, only patients with adenocarcinoma in the UFT group had a significantly better survival rate than patients with other cell types [11]. It has been demonstrated that adjuvant therapy is not effective in all patients with stage I disease; it is effective only in specific subsets of patients. It is important to select a subgroup of patients with stage I disease who might benefit from adjuvant therapy, and for this reason investigators have attempted to pinpoint factors that predict poor prognosis through analysis of clinical and histopathologic factors [12-15]. Therefore, the goals of this study were to identify these prognostic factors and to
Table 4 Univariate and multivariate analysis of diseasefree survival

\begin{tabular}{llcc}
\hline Factors & Variables & $\begin{array}{l}\text { Univariate } \\
\text { analysis }\end{array}$ & $\begin{array}{l}\text { Multivariate } \\
\text { analysis }\end{array}$ \\
\hline T stage & $1 / 2$ & 0.037 & $>0.05$ \\
Tumor size & $\leq 3 \mathrm{~cm} />3 \mathrm{~cm}$ & $>0.05$ & $>0.05$ \\
Necrosis & Absent/present & 0.010 & 0.009 \\
Differentiation & Well/mod/poor & 0.021 & $>0.05$ \\
Vascular invasion & Absent/present & $>0.05$ & $>0.05$ \\
Lymphatic vessel & Absent/present & 0.017 & 0.04 \\
invasion & Absent/present & $>0.05$ & $>0.05$ \\
Visceral pleural invasion & Loss/preserved & $>0.05$ & $>0.05$ \\
p16 & Loss/preserved & $>0.05$ & $>0.05$ \\
Rb & Normal/overexpressed & $>0.05$ & $>0.05$ \\
Cyclin D & Loss/preserved & 0.006 & 0.026 \\
E-cadherin & Negative/positive & 0.046 & 0.010 \\
p53 & 1\&2/3\&4 & $>0.05$ & $>0.05$ \\
Glut-1 & Low LI/high LI & $>0.05$ & 0.031 \\
Ki-67 & Normal/overexpressed & 0.041 & $>0.05$ \\
EGFR & & & \\
\hline
\end{tabular}



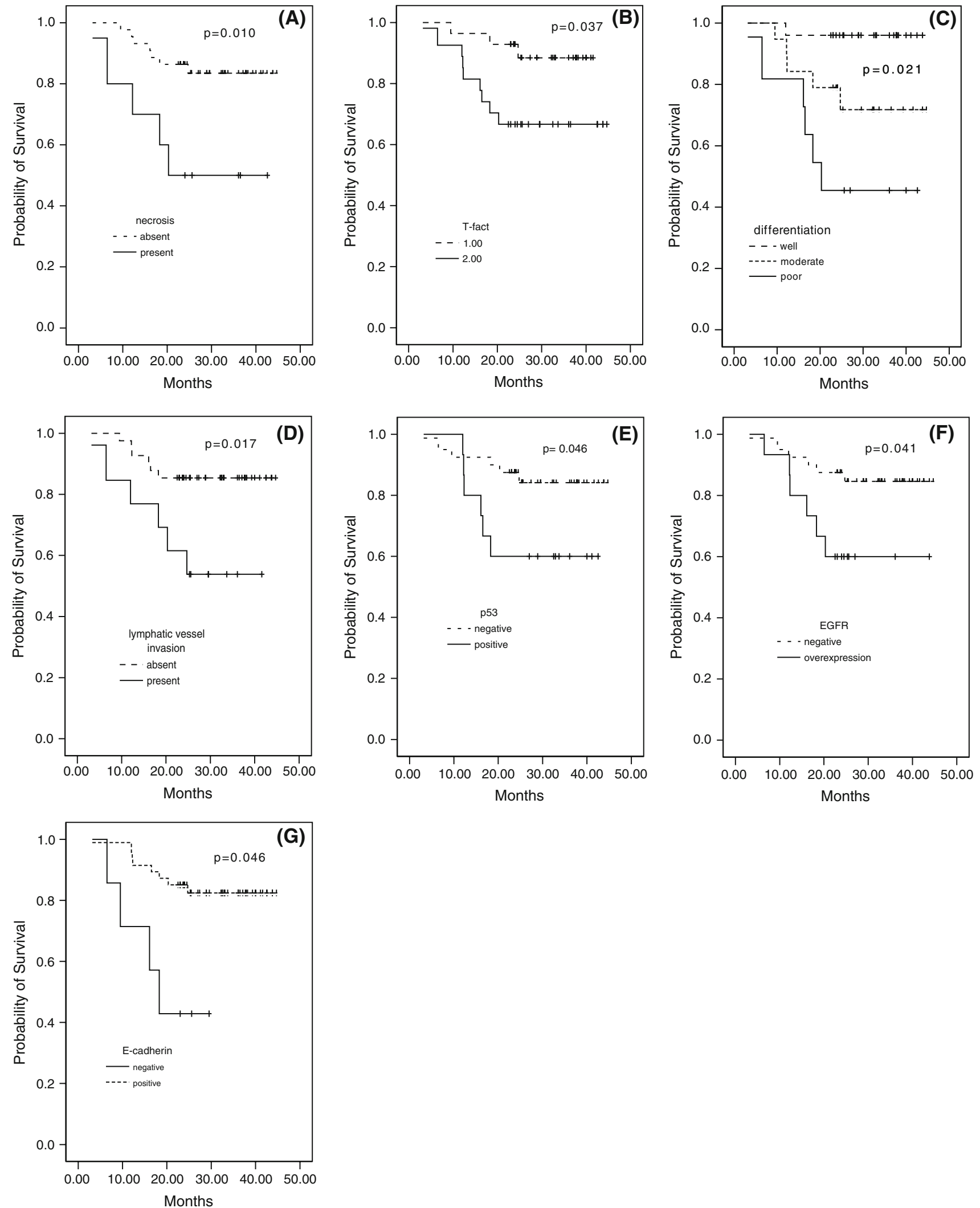

Fig. 1 Kaplan-Meier analysis of disease-free survival for (a) necrosis, (b) T-factor, (c) differentiation, (d) lymphatic vessel invasion, (e) p53, (f) EGFR, and (g) E-cadherin 
Fig. 2 Analysis of disease-free survival (a) according to the involvement of the four prognostic factors that were significant in both univariate and multivariate analysis and (b) according to the risk group: the low-risk group has fewer than two risk factors and the high-risk group has two or more risk factors

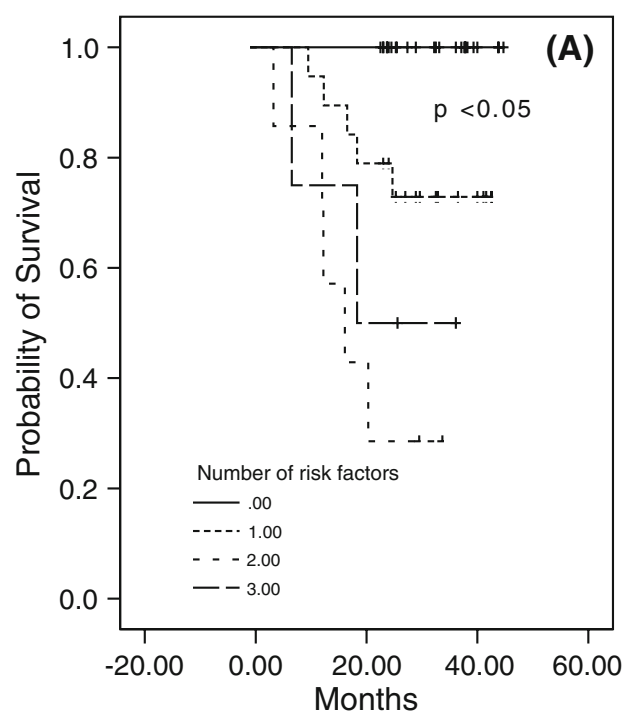

evaluate the association between histopathologic and molecular markers.

A multitude of histopathologic factors associated with survival have been described in the literature through retrospective studies. Harpole et al. [5] endeavored to define risk factors associated with recurrence and death in 289 resected stage I lung cancer patients; vascular invasion, visceral pleural invasion, high mitotic index, and tumor size greater than $3 \mathrm{~cm}$ were identified. The degree of differentiation of the primary tumor is known to be a significant prognostic factor and poorly differentiated tumors are defined as negative prognostic factors in patients with stage I NSCLC [16]. In this study, poorly differentiated tumors were related to high-grade Glut-1 expression and a high $\mathrm{Ki}-67 \mathrm{LI}$, which indicates that a poorly differentiated tumor has high proliferative activity and requires more glucose uptake for growth. The tumor growth rate was higher in less-differentiated tumors, and necrosis was found to be related inversely to differentiation and associated with tumor size [9]. In the present study, patients with tumors with necrosis had a significantly shorter time to recurrence than patients without necrosis.

Peritumoral lymphatic vessel invasion has been demonstrated to have prognostic significance for local and distant recurrence [17]. The first step of lymph node metastasis is cancer cell invasion of the lymphatic vessels within the tumors; therefore, tumors that have lymphatic vessels invasion of the tumour cells have a potential risk of local recurrence [18]. Although data based on clinical and histopathologic descriptions aids in dividing patients into risk groups, there is much variation in the interpretation of these qualitative variables. Therefore, many molecular biological markers have been studied in lung cancer to determine their prognostic importance. Gene copy number and mutation analyses are now being explored in prospective clinical studies in enriched populations. These assays are very promising, but they are costly and are not widely available. In contrast, protein expression studies by immunohistochemistry (IHC) are rapid, reproducible, and routinely performed in many hospitals. During the last decade, the aggressive phenotypes of lung cancer have been characterized by a number of molecular abnormalities, so multiple algorithms were created based on relative protein expression profiles using up to 15 factors with possible significance. These studies showed that the primary factors associated with prognosis were $\mathrm{p} 53$, factor VIII, Her2-neu, CD44, and Rb [19]. Considering these results, markers that represent five separate oncogenic pathways were chosen for this study: growth factor (EGFR), cell cycle-related factors ( $\mathrm{p} 16$, cyclinD1, Rb, p53), metastatic adhesion factor (E-cadherin), metabolic factor (Glut-1), and proliferation factor (Ki-67). With both univariate and multivariate analysis, our results showed that p53 and E-cadherin are significant prognostic factors.

Because p53 is a tumor suppressor gene that can induce temporary growth arrest and DNA repair or apoptosis in response to potentially oncogenic cellular stress such as DNA damage, the impairment of cell growth control results in uncontrolled cell proliferation [20]. However, the relationship between p53 expression and prognosis remains unclear, even though abnormal p53 protein expression and p53 gene mutation have been extensively investigated for their prognostic value in early-stage resected NSCLC patients. Some investigators have demonstrated that p53 overexpression is an independent prognostic factor in nodenegative NSCLC, although there is no difference in clinical outcome between p53-positive and p53-negative tumors in node-positive cases [21]. The frequency of p53 mutation or 
protein expression is consistently higher in squamous cell carcinoma than in adenocarcinoma, but the impact appears greater in the latter $[22,23]$.

E-cadherin and catenins are components of the adherens junction protein, with crucial roles in maintaining intercellular junctions in epithelial cells; reduced E-cadherin expression could potentially affect tumor differentiation, metastasis, and prognosis [24, 25]. Therefore, it might be associated with a pathologic factor of metastatic activity, and in this study, E-cadherin expresssion is associated with lymphatic vessel invasion $(p=0.021)$. Although the number of patients who demonstrated loss of E-cadherin is small, it still represents a statistically significant prognostic factor.

D'Amico et al. [9], using the prognostic risk model for survival, demonstrated significantly decreased survival according to the total number of factors involved with multivariable analysis of the entire panel of molecular markers. Lu et al. [26] reported similar results. In their study, they revealed that two factors were significant negative prognostic factors and defined the patients with two factors as high-risk. A log-rank test assessing the difference in survival was statistically significant for both disease-specific survival and overall survival [26].

This study had limitations. First, immunohistochemical scoring is at best a semiquantitative exercise for which no standard criteria have yet been proposed or adopted. The scoring methods that have been used included estimating the grade of staining intensity, percentage of tumor cells stained, cellular localization of the antigen, and systems that combined these parameters. Second, more importantly, there is confusion in the method of determining cutoffs for dichotomizing scores in log-rank tests. Cutoffs are often arbitrary and are sometimes selected to obtain the desired effect using the minimum $p$-value approach. Therefore, different antibodies and grading systems should also be taken into account; the counting of positive tumor cells and grading of staining intensity may be subject to interobserver variability, which should be evaluated. Third, due to the short follow-up period, some markers, if any, that influence late recurrence would not be prognostic factors. However, in lung cancer, most recurrence occurs within 2 years, and 3-year follow-up periods are considered acceptable. Fourth, this study has a small number of patients. As mentioned above, lung cancer is a heterogeneous disease and there is much confusion about the evaluation of molecular markers in all stages and every cell type of lung cancer. Therefore, even though it resulted in a smaller number of patients, the study population was intentionally confined to a homogeneous group of resected stage I adenocarcinoma patients. Based on these results, it is possible to tailor individualized therapy to stage I lung cancer. Despite the lack of results to support adjuvant chemotherapy in stage I diseases outside a clinical trial setting, it is probably reasonable to consider the possibility of adjuvant systemic chemotherapy in individual healthy younger patients whose resected tumors exhibit poor prognostic histologic findings and who have associated molecular markers such as lymphatic vessel invasion, necrosis, p53 overexpression, and E-cadherin loss.

Acknowledgment This work was supported by grant No (02-2006030) from the Seoul National University Bundang Hospital (SNUBH) Research Fund.

\section{References}

1. Travis WD, Colby TV, Corrin B (1999) Histological typing of lung and pleural tumors, 3rd edn. OMS Springer, Heidelberg

2. Mountain CF, Lukeman JM, Hammar SP (1987) Lung cancer classification: the relationship of disease extent and cell type to survival in a clinical trials population. J Surg Oncol 35:147-156. doi:10.1002/jso.2930350302

3. Nesbitt JC, Putnam JB Jr, Walsh GL, Roth JA, Mountain CF (1995) Survival in early-stage non-small cell lung cancer. Ann Thorac Surg 60:466-472. doi:10.1016/0003-4975(95)00169-L

4. Cox G, Jones JL, Andi A, Waller DA, O'Byme KJ (2001) A biological staging model for operable non-small cell lung cancer. Thorax 56:561-566. doi:10.1136/thorax.56.7.561

5. Harpole DH Jr, Richards WG, Herndon JEII, Sugarbaker DJ (1996) Angiogenesis and molecular biologic substaging in patients with stage I non-small cell lung cancer. Ann Thorac Surg 61:1470-1476. doi:10.1016/0003-4975(96)00104-X

6. Strauss GM, Kwiatowski DJ, Harpole DH, Lynch TJ, Skarin AT, Sugarbaker DJ (1995) Molecular and pathologic marker in stage I non-small cell lung carcinoma of the lung. J Clin Oncol 13:12651279

7. Duarte IG, Bufkin BL, Pennington MF, Gal AA, Cohen C, Kosinski AS, Mansour KA, Miller JI (1998) Angiogenesis a predictor of survival after surgical resection for stage I non-small cell lung cancer. J Thorac Cardiovasc Surg 115:652-659. doi: 10.1016/S0022-5223(98)70331-9

8. Quinlan DC, Davidson AG, Summers CL, Warden HE, Doshi HM (1992) Accumulation of p53 proteins correlates with a poor prognosis in human lung cancer. Cancer Res 52:4828-4831

9. Poleri C, Morero JL, Nieva B, Vázquez MF, Rodríguez C, de Titto E, Rosenberg M (2003) Risk of recurrence in patients with surgically resected stage I non-small cell lung carcinoma: histopathologic and immunohistochemical analysis. Chest 123:18581867. doi:10.1378/chest.123.6.1858

10. Strauss GM, Herndon J, Maddaus MA (2006) Adjuvant chemotherapy in stage IB NSCLC: update of CALGB protocol 9633. ASCO Annual Meeting Proceedings. J Clin Oncol 24:7007

11. Kato H, Ichinose Y, Ohta M, Hata E, Tsubota N, Tada H, Watanabe Y, Wada H, Tsuboi M, Hamajima N, Ohta M, Japan Lung Cancer Research Group on Postsurgical Adjuvant Chemotherapy (2004) A randomized trial of adjuvant chemotherapy with uraciltegafur for adenocarcinoma of the lung. N Engl J Med 350: 1713-1721. doi:10.1056/NEJMoa032792

12. Macchiarini P, Fontanini G, Hardin MJ, Chuanchieh H, Bigini D, Vignati S, Pingitore R, Angeletti CA (1993) Blood vessel invasion by tumor cells predicts recurrence in completely resected T1NOM0 non-small cell lung cancer. J Thorac Cardiovasc Surg 106:80-89

13. Thomas PA, Rubinstein L (1990) Cancer recurrence after resection: T1 N0 non-small cell lung cancer. Ann Thorac Surg 49: 242-247 
14. Takise A, Kodama A, Shimosato Y, Watanabe S, Suemasu K (1988) Histologic prognostic factors in adenocarcinoma of the lung periphery less than $2 \mathrm{~cm}$ in diameter. Cancer 61:2083-2088. doi:10.1002/1097-0142(19880515)61:10<2083::AIDCNCR2820611025>3.0.CO;2-U

15. Pairolero PC, Williams DE, Bergstralh EJ, Bernatz PE, Payne WS (1984) Postsurgical stage I bronchogenic carcinoma: morbid implications of recurrent disease. Ann Thorac Surg 38:331-336

16. Kobayashi N, Toyooka S, Soh J, Ichimura K, Yanai H, Suehisa H, Ichihara S, Yamane M, Aoe M, Sano Y, Date H (2007) Risk factors for recurrence and unfavorable prognosis in patients with stage I non-small cell lung cancer and a tumor diameter of $20 \mathrm{~mm}$ or less. J Thorac Oncol 2:808-812. doi:10.1097/JTO.0b013e3 $1814617 \mathrm{c} 7$

17. Fujisawa T, Yamaguchi Y, Saitoh Y, Hiroshima K, Ohwada H (1995) Blood and lymphatic vessel invasion as prognostic factors for patients with primary resected nonsmall cell carcinoma of the lung with intrapulmonary metastases. Cancer 76:2464-2470. doi:10.1002/1097-0142(19951215)76:12<2464::AID-

CNCR2820761210>3.0.CO;2-U

18. Ichinose $\mathrm{Y}$, Yano $\mathrm{T}$, Yokoyama $\mathrm{H}$, Inoue $\mathrm{T}$, Asoh $\mathrm{H}$, Katsuda $\mathrm{Y}$ (1994) The correlation between tumor size and lymphatic vessel invasion in resected peripheral stage I non-small cell lung cancer. J Thorac Cardiovasc Surg 108:684-686

19. D'Amico TA, Massey M, Herndon JE II, Moore MB, Harpole DH (1999) A biologic risk model for stage I lung cancer: immunohistochemical analysis of 408 patients with the use of ten molecular markers. J Thorac Cardiovasc Surg 117:736-743. doi: 10.1016/S0022-5223(99)70294-1

20. Lane D (1992) p53, guardian of the genome. Nature 358:15-16. doi: $10.1038 / 358015 \mathrm{a} 0$
21. Dalquen P, Sauter G, Torhorst J, Schultheiss E, Jordan P, Lehmann S, Solèr M, Stulz P, Mihatsch MJ, Gudat F (1996) Nuclear p53 overexpression is an independent prognostic parameter in node-negative non-small cell lung carcinoma. J Pathol 178:5358. doi:10.1002/(SICI)1096-9896(199601)178:1<53::AID-PATH $415>3.0 . \mathrm{CO} ; 2-\mathrm{T}$

22. Mitsudomi T, Hamajima N, Ogawa M, Takahashi T (2000) Prognostic significance of $\mathrm{p} 53$ alterations in patients with nonsmall cell lung cancer: A meta-analysis. Clin Cancer Res 6:40554063

23. Steels E, Paesmans M, Berghmans T, Branle F, Lemaitre F, Mascaux C, Meert AP, Vallot F, Lafitte JJ, Sculier JP (2001) Role of p53 as a prognostic factor for survival in lung cancer: as systemic review of the literature with a meta-analysis. Eur Respir J 18:705-719. doi:10.1183/09031936.01.00062201

24. Liu D, Huang C, Kameyama K, Hayashi E, Yamauchi A, Kobayashi S, Yokomise H (2001) E-cadherin expression associated with differentiation and prognosis in patients with non-small cell lung cancer. Ann Thorac Surg 71:949-955. doi:10.1016/S00034975(00)02545-5

25. Kase S, Sugio K, Yamazaki K, Okamoto T, Yano T, Sugimachi K (2000) Expression of E-cadherin and b-catenin in human nonsmall cell lung cancer and the clinical significance. Clin Cancer Res 6:4789-4796

26. Lu C, Soria JC, Tang X, Xu XC, Wanag L, Mao L, Lotan R, Kemp B, Bekele BN, Feng L, Hong WK, Khuri FR (2004) Prognostic factors in resected stage I non-small cell lung cancer: a Multivariate analysis of six molecular markers. J Clin Oncol 22:4575-4583. doi:10.1200/JCO.2004.01.091 